\title{
Impacto de un programa de equitación terapéutica en la mejora de la psicomotricidad de niños autistas
}

\section{Impact of therapeutic riding program in improving motor skills of autistic children}

\author{
Sonia Bouzo-González*, Margarita R. Pino-Juste** \\ *Profesora-Tutora CA UNED Pontevedra, **Profesora Titular Universidade de Vigo
}

\begin{abstract}
Resumen
En este texto se presentan los resultados de un estudio descriptivo, de corte cualitativo, en el cual hemos utilizado como método de investigación el estudio de caso, con el objetivo final de demostrar los múltiples beneficios que aporta la Equitación Terapéutica con niños autistas.

Las técnicas de registro utilizadas han sido la observación, un cuestionario y un diario de campo.

Entre los resultados más destacados señalamos ventajas como la mejora en el equilibrio y la postura. Visibles mejoras tanto en la motricidad fina como gruesa, y una relajación muscular óptima que favorecía la eliminación paulatina de las estereotipias presentadas por nuestros usuarios.

Palabras clave: Hipoterapia, Equitación Terapéutica, Autismo y psicomotricidad.
\end{abstract}

\begin{abstract}
In this paper are presented the results of a descriptive study of qualitative cutting, in which we have used as a method of research case study, with the ultimate goal of demonstrating the multiple benefits that provides horse riding therapy with autistic children. Recording techniques used have been the observation, a questionnaire and a field journal. Among the most outstanding results noted benefits such as improvement in balance and posture. Visible improvement in fine motor as thick, and an optimal muscle relaxation which favored the gradual elimination of the stereotypic movements submitted by our users.

Key words: hippotherapy, therapeutic riding, autism and psychomotor skills.
\end{abstract}

\section{Introducción}

En los últimos años ha aumentado considerablemente el interés por las intervenciones con asistencia animal como una opción terapéutica para un amplio rango de trastornos del desarrollo y otras discapacidades. La terapia asistida con animales, es decir, aquella que utiliza animales para implementar un tratamiento, ha demostrado tener beneficios cognitivos, psicológicos y sociales (Fine, 2006). Además, este tipo de terapias ha probado tener efectos positivos en diversos factores fisiológicos como pueden ser la disminución de la presión arterial y las pulsaciones o la bajada de los niveles de ansiedad (Morrison, 2007). En concreto, la hipoterapia ha obtenido resultados positivos en diversas intervenciones llevadas a cabo con niños autistas y con otros tipos de discapacidades.
La etimología del autismo procede del griego donde autos significa "consigo mismo". Y es, precisamente, la dificultad de aceptar al otro y su punto de vista lo que define al autismo. Kanner (1943) fue el primer psiquiatra infantil que denominó la patología por lo que el autismo clásico se denomina a veces "autismo de Kanner".

La prevalencia del autismo ha variado enormemente del 4 por 10.000 hace 40 años al 1 por ciento actual, lo que implica que se ha multiplicado por 25. Esto es debido, en parte, a un cambio del concepto "autista": del diagnóstico categórico se ha pasado a considerar el autismo como un trastorno de espectro, por lo que se ha ampliado el abanico a los casos leves que también causan sufrimiento y deben ser tratados. En este aumento de la prevalencia también han influido otros factores como el hecho de que actualmente se diagnostique mejor ya que terapeutas, médicos generales y pediatras, psicólogos y psiquiatras infantiles saben lo que es el autismo; o la inclusión de nuevos subgrupos además del autismo clásico.

Actualmente, los científicos no saben exactamente qué origina el autismo y debido a que se trata de un trastorno complejo y a que no hay dos personas que manifiesten la enfermedad de manera exactamente igual, se piensa que es probable que tenga muchas causas (NICHD, 2005). Podemos concluir que se caracteriza por una alteración en la relación social recíproca, en la comunicación, el lenguaje y la imaginación, por conductas rígidas, intereses y actividades muy restringidas y estereotipadas. Con frecuencia estos síntomas se acompañan de comportamientos anormales, estereotipias motoras y obsesiones hacia determinados objetos o sucesos, pudiendo aparecer también conductas auto y heteroagresivas (Vega, 2005).

El objetivo del presente estudio es contribuir a establecer los beneficios de la equinoterapia como tratamiento para niños autistas en el área del desarrollo psicomotriz, mediante el diseño y la realización de una intervención de hipoterapia y equitación terapéutica.

La equinoterapia se puede considerar una terapia integral, que no solo cumple funciones fisioterapéuticas sino que también ofrece amplios beneficios en el área psicológica (Murphy et al., 2008). Se ha ganado un importante lugar como terapia alternativa por sus objetivos tanto en el área de la fisioterapia como de la psicoterapia. 
Siguiendo a Gross (2006) con el término genérico "equinoterapia" se denominan diversas técnicas orientadas al tratamiento de personas con discapacidad, en las que uno de sus elementos centrales es el caballo. El contacto con el caballo proporciona múltiples sensaciones que influyen positivamente en los ámbitos social, sensorial y motor. El paso de este animal simula a la perfección el deambular humano (Benda, McGibbon \& Grant, 2003; McPhail, 2006; Snider, et al. 2007)

Podemos hablar entonces de la división aceptada a nivel internacional de la equinoterapia en tres áreas: Hipoterapia, Monta terapéutica y volteo, y Equitación como deporte para discapacitados (Gross, 2006).

La equinoterapia como término global de las tres áreas abarca la integración de cuatro ámbitos profesionales diferentes: la medicina, la psicología, la pedagogía y el deporte. El área médica desempeña un papel predominante en la Hipoterapia, pues la monta a caballo se utiliza en forma de fisioterapia, indicada para pacientes con disfunciones neuromotoras de origen neurológico, traumático o degenerativo. En el trabajo terapéutico se utiliza, en primer lugar, la hipoterapia. Luego, cuando el paciente va evolucionando en su tratamiento, se pasa a la siguiente etapa que es la monta terapéutica, y finalmente continúa la equitación como deporte para discapacitados, o "equitación adaptada".

\section{Método}

Se trata de una investigación evaluativa de caso grupal desde un enfoque interpretativo-descriptivo con medidas pretest-postest utilizando un método de investigaciónacción.

En el presente trabajo la autora de la investigación ha participado activamente en la intervención como planificadora y orientadora de las sesiones de hipoterapia y equitación terapéutica. Apoyada por un valioso Equipo Interdisciplinar (formado por tres monitores de equitación, dos fisioterapeutas, la autora (orientadora) debidamente formadas en dicha terapia rehabilitadora, y a la par conocedoras de las particularidades del problema que presentaban nuestros sujetos objeto de estudio- así como varios voluntarios) han ayudado a los participantes a mejorar su desarrollo psicomotriz según los objetivos fijados, mientras se llevaba a cabo un programa de equitación terapéutica adaptado a las peculiaridades de cada uno de los usuarios.

Como enfoque o paradigma se ha escogido el interpretativo-descriptivo ya que por sus características es especialmente adecuado a los estudios de caso. Hablamos de un estudio cuya realización tiene lugar en un ambiente natural, puesto que los fenómenos deben estar en sus contextos para ser comprendidos; el sujeto humano es el instrumento de investigación por antonomasia. Se ha utilizado el conocimiento tácito así como métodos cualitativos. El análisis de datos ha sido de carácter inductivo con una teoría fundamentada y enraizada y el informe final presenta la forma de estudio de casos: recogiendo, entre otros aspectos, una descripción completa del contexto y del papel del investigador en el proceso de comunicación con los sujetos. Se aporta una interpretación ideográfica, puesto que las interpretaciones se realizan remitiéndose a la particularidad de cada caso analizado y dependen del contexto concreto y de las relaciones establecidas entre el investigador y los informantes, y finalmente destacar que se parte de unos criterios especiales para la confiabilidad.

Se ha elegido una metodología de estudio de caso evaluativo de orientación etnográfica con la pretensión de describir, analizar y comprender cómo las acciones humanas se relacionan en el contexto social y educativo donde ocurren. En consecuencia, la unidad de análisis ha de examinarse en su entorno educativo, social y cultural para poder llegar a emitir juicios sobre la realidad objeto de estudio.

La elección del estudio de caso se apoyó en tres razones: su carácter crítico, es decir, en la medida que permite confirmar, cambiar, modificar o ampliar el conocimiento sobre el objeto de estudio; su carácter extremo o unicidad, pues parte de una situación que tiene un carácter específico y peculiar; y, su carácter revelador que permite observar y analizar un fenómeno o hecho particular relativamente desconocido en la investigación educativa y sobre el cual pueden realizarse aportaciones de enorme relevancia.

\section{Participantes}

Nuestra muestra de estudio está compuesta por cinco sujetos autistas muy diferentes y a los cuales describiremos a continuación:

EMA: varón de 31 años, sin lenguaje oral establecido, se comunica mediante expresiones ecolálicas sin funcionalidad. Presenta problemas de lateralidad y coordinación. Presenta episodios de autolesiones, sobre todo cuando se encuentra ante situaciones nuevas que le generan ansiedad y estrés, así como diferentes estereotipias motoras como aleteo de brazos.

JRS: varón de 14 años, no presenta comunicación con función declarativa aunque realiza actividades de pedir señalando aquello que desea. Presenta defensa táctil a mantener contacto físico con personas nuevas $\mathrm{e}$ irritabilidad ante los cambios de rutina y ambientes nuevos. Los problemas más destacados de JRS se dan en el área de los comportamientos estereotípicos movimiento lateral con la cabeza, aleteo, balanceos, giros, pone las manos a ambos lados de la cara y a la altura de los ojos como si estuviera meditando, emisión de sonidos autoestimulatorios y autolesiones (a veces se muerde o intenta morder al terapeuta o cuidador). En lo que se refiere a su patrón motor JRS camina encorvado con los hombros hacia delante y los brazos colgando, arrastra los pies al caminar y su tono muscular y forma física están muy debilitados, presenta un ligero sobrepeso para su estatura y edad.

MAGS: varón de 19 años, no presenta lenguaje oral funcional, se comunica mediante gestos cuando quiere conseguir algo (lo señala o te coge de la mano y te lleva) apenas mantiene contacto ocular con las demás personas que le rodean. Respecto a su conducta motora y funcional cabe destacar que el joven camina de forma normal 
aunque presenta movimientos de balanceo, oscilaciones de cuerpo, aleteo de brazos, etc. de forma regular. MAGS se muestra ansioso o tenso con frecuencia. Grita con frecuencia y tiene rabietas.

ERT: varón de 13 años, no presenta lenguaje oral funcional, solo emite sonidos sin finalidad comunicativa. No presenta resistencia a pequeños cambios en el ambiente pero insiste en que exista una repetición exacta de las rutinas diarias. Cuando está nervioso, contento o se aburre aparecen movimientos de balanceo, oscilaciones de cuerpo, aleteo de brazos, etc. Su patrón de deambulación es bastante deficitario, arrastra los pies cuando camina, y su tono muscular está debilitado además de presentar un ligero sobrepeso.

LNS: hembra de 8 años, LNS no habla pero entiende las frases sencillas y parece comprender gestos como acudir cuando se la llama con la mano o cuando se le pide que guarde silencio con el dedo sobre los labios. No muestra resistencia o ansiedad a los cambios ni necesita una repetición exacta de las rutinas diarias. Suele retorcerse los dedos. Respecto a su conducta motora y postural cabe destacar que presenta movimientos de balanceo, aleteo de brazos, saltos, gestos y giros y adopta posturas raras con las manos y con el cuerpo. Su patrón deambulatorio presenta gran dependencia física (necesita ir cogida de la mano de un cuidador para caminar) y debilidad muscular así como un escaso desarrollo psicomotriz tanto a nivel fino como grueso.

\section{Procedimiento}

El estudio se ha llevado a cabo a lo largo de un curso escolar, nueve meses, con una periodicidad de cuatro sesiones al mes, una sesión por semana, y en cada una de las cuales se han realizado una serie de mediciones y observaciones para dar paso a un exhaustivo análisis de datos final que diese como resultado el demostrar las múltiples ventajas de dicha técnica en el desarrollo psicomotor de estos niños.

Hemos trabajado buscando mejorar y aumentar su capacidad de relajación para reducir la tensión muscular y favorecer una respiración más regular y cómoda, con la consiguiente modificación de los ritmos eléctricos del cerebro. Mediante la relajación se consiguen beneficios tanto físicos como psicológicos. En niños autistas, la relajación se utiliza para distender el organismo y reducir el estrés muscular, canalizando la energía y ajustando el nivel de activación, de forma que se consigue alcanzar un estado de bienestar y tranquilidad. La relajación contribuye a disminuir los niveles de ansiedad, angustia y pánico y consecuentemente reduce las estereotipias. A través del movimiento del caballo se consigue la relajación física requerida para eliminar estereotipias y mejorar el control y la corrección postural, el tono muscular y la coordinación; y en un segundo plano buscábamos la relajación psíquica necesaria para aumentar la motivación, la confianza y la autonomía que ayudan en la eliminación y corrección de conductas autoagresivas y heteroagresivas.

Otro de nuestros objetivos era el de fomentar la mejora de las actitudes posturales que conllevan mantener equilibrado el cuerpo. Los niños autistas suelen presentar hipotonía generalizada con paratonías frecuentes así como inestabilidad y conductas de agitación frecuentes. A través de la equinoterapia se fortalece la musculatura y se trabaja el equilibrio y la postura, para mejorar la motricidad general.

Pretendiamos que nuestros pacientes fuesen capaces de asimilar una serie de habilidades y destrezas que les permitiesen un mínimo dominio, en la medida de lo posible, de la técnica ecuestre. De esta forma los niños que participaron en el programa además de mejorar en el área de psicomotricidad también aprendían una nueva habilidad de ocio que les podría resultar muy gratificante a lo largo de su vida.

En la siguiente figura podemos observar la relación de objetivos y competencias del Programa, en cuanto al desarrollo de habilidades motoras.

\begin{tabular}{|c|c|}
\hline Objetivo & Competencias \\
\hline $\begin{array}{l}\text { Aumentar la capacidad de } \\
\text { relajación }\end{array}$ & $\begin{array}{l}\text { Capacidad de relajación muscular } \\
\text { Correcta integración sensorial } \\
\text { Reducción de las estereotipias }\end{array}$ \\
\hline $\begin{array}{lll}\text { Mejorar las actitudes } \\
\text { posturales }\end{array}$ & $\begin{array}{l}\text { Mejorar la postura y el equilibrio } \\
\text { Fortalecimiento de la musculatura } \\
\text { Motricidad general (fina y gruesa) }\end{array}$ \\
\hline $\begin{array}{l}\text { Mejorar la técnica de } \\
\text { equitación }\end{array}$ & $\begin{array}{l}\text { Mejorar la postura y el equilibrio } \\
\text { Fortalecimiento de la musculatura } \\
\text { Motricidad general (fina y gruesa) }\end{array}$ \\
\hline
\end{tabular}

Figura 1. Relación entre los objetivos y las competencias del Programa de Hipoterapia Adaptado. Fuente: Elaboración propia.

Las sesiones se han desarrollado en pista cubierta, en pista descubierta y en paseos por el campo. La pista cubierta está especialmente indicada para aquellos casos en los que la variabilidad del entorno puede suponer un grave trastorno para la tranquilidad del usuario e influir negativamente en sus reacciones y adaptación a la terapia. La pista descubierta se utiliza con los usuarios que tienen o han alcanzado tolerancia a los cambios externos. Y por último los paseos por el campo favorecen la integración sensorial una vez que el usuario se ha adaptado a las sesiones.

La dinámica de las sesiones solía seguir unas pautas, se iniciaba mediante el contacto del usuario con el caballo en una actividad que consistía en la higiene y preparación del caballo; tras esta toma de contacto el sujeto "monta" al animal, dependiendo de su grado de discapacidad física se le facilitan ayudas y apoyos, pero siempre promoviendo su mayor grado de independencia y autonomía; durante la actividad de monta terapéutica se realizan ejercicios de psicomotricidad, corrección postural y mejora del tono muscular, interrelacionados siempre con ejercicios de atención, comunicación y relación con otros sujetos (compañeros de terapia, terapeuta, caballo).

\section{Instrumentos}

Las técnicas de investigación empleadas en la recogida de datos del presente estudio han sido las Entrevistas realizadas a las familias, a la dirección del Centro Educativo y a los diferentes Terapeutas que trabajaban 
con los niños sincrónicamente, así como la obtención de datos a través de un exhaustivo Análisis de Documentos (anamnesis, valoraciones y diagnósticos médicos, etc.) La Observación Participante (mediante la implementación de una serie de Fichas de Registro Observacional Diario, que han sido cubiertas a lo largo de todas y cada una de las sesiones). Un Cuestionario (Con la finalidad de determinar el impacto del programa, a través de un pretest-postest diseñado con el objetivo de conocer el desarrollo del área motora de nuestros participantes al principio y final de la intervención), este instrumento se ha elaborado en base a las características de los TEA establecidas por el DSM-IV, adaptándolas a las actividades hipoterapéuticas que se han llevado a cabo en el programa. Y finalmente un Diario de Campo donde se reflejan los diferentes comentarios y aportaciones de los distintos miembros del Equipo Multidisciplinar que han intervenido en dicho Programa.

\section{Resultados}

En la equinoterapia como en cualquier otro tipo de terapia, la evaluación es indispensable, sobre todo su realización de forma periódica, como medio de retroalimentación. Las evaluaciones iniciales del paciente nos han proporcionado una indicación precisa de los problemas y limitaciones que presentaban a nivel de desarrollo psicomotor. Las evaluaciones posteriores tenían como misión indicar al terapeuta la eficiencia de sus estrategias, y nos han servido de guía para la generación de los nuevos objetivos terapéuticos y la metodología a seguir.

Como recordaremos el primer objetivo al que hacíamos referencia era aumentar la capacidad de relajación. En relación con este objetivo podemos afirmar que nuestros usuarios han mejorado de forma sensible su capacidad de relajación muscular, aumentando su integración sensorial y reduciendo significativamente sus estereotipias.

En la tabla siguiente podemos comprobar como todos los ítems evaluados en el cuestionario en relación a este objetivo han mejorado, siendo la mejora total de un punto.

Tabla 1.

Media de las puntuaciones adquiridas en desarrollo motor

\begin{tabular}{lcc}
\hline & $\begin{array}{c}\text { Media } \\
\text { Pre }\end{array}$ & $\begin{array}{c}\text { Media } \\
\text { Post }\end{array}$ \\
\hline Coordinación dinámica & 1,8 & 2,6 \\
Disociación motriz & 1,8 & 2,6 \\
Coordinación visomotriz & 1,2 & 2,6 \\
Orientación espacial & 1,4 & 2,4 \\
Estructuración temporal & 1,6 & 2,6 \\
Total & 1,56 & 2,56 \\
\hline
\end{tabular}

Fuente: Elaboración propia.

Como puede verse en la tabla en todas las áreas medidas en el cuestionario se obtuvo una mejora en el conjunto de los casos. Cabe destacar la mejora en la coordinación visomotriz que fue bastante baja en el pretest y considerablemente alta en el post-test.
Tabla 2

\begin{tabular}{lcc} 
Media de las puntuaciones adquiridas & en desarrollo motor \\
\hline & $\begin{array}{c}\text { Media } \\
\text { Pre }\end{array}$ & $\begin{array}{c}\text { Media } \\
\text { Post }\end{array}$ \\
\hline & & \\
\hline Control tónico-postural & 1,4 & 2,4 \\
Equilibrio & 1,8 & 2,4 \\
Lateralización & 1 & 2 \\
Total & 1,4 & 2,27 \\
\hline
\end{tabular}

Fuente: Elaboración propia.

Las aciniuaues yue inlas iavorecieron la consecucion de este objetivo fueron los "paseos por el campo". A pesar del problema que suelen presentar los autistas con los ruidos, el paseo contribuía a reducir los estados de ansiedad, angustia o pánico de los participantes ya que "al poco tiempo de salir al campo observamos como los participantes se tranquilizaban con la cadencia del caballo al caminar" (Según datos del Diario de Campo, Monitora de Equitación).

En cuanto al objetivo de mejorar las actitudes posturales ha sido conseguido como veremos hasta en los casos más difíciles. Todos los participantes han mejorado su postura y el equilibrio tanto sobre el caballo como pie a tierra, han fortalecido su musculatura y han conseguido una mejora en la motricidad fina y gruesa. Como podemos ver en la siguiente tabla, en todos los ítems medidos los participantes han conseguido beneficios significativos.

Como se recoge en el diario de campo, en prácticamente todos los casos y sesiones se consiguió trabajar el control postural, a algunos sujetos había que recordárselo más a menudo pero las indicaciones verbales y el contacto visual eran suficientes. " $L a$ hipoterapia les ha ayudado a fortalecer su musculatura y cabe destacar la mejoría en la motricidad gruesa que han desarrollado para conseguir guiar al caballo, pararlo y emprender la marcha de nuevo" (Diario de Campo, Monitora de Equitación). "Tras las sesiones, su equilibrio en bipedestación se ha visto favorecido nada más bajar del caballo, los sujetos caminaban más erguidos, y con mayor rectitud y estabilidad" (Diario de Campo, Fisioterapeuta). Pero es en la motricidad fina donde más avances se han conseguido ya que todos han logrado coger las riendas con las dos manos sin levantarlas continuamente (estereotipias de EMA y ERT), morderse las manos (JRS), o entrelazar los dedos y llevarse las manos a la boca continuamente (LNS).

Según los parámetros evaluados y observados mediante el Registro Observacional dentro de los subapartados de la Técnica Utilizada y el desarrollo de la Actividad Ecuestre, destacar que "durante el programa se consiguió que practicasen el galope (aire superior y más veloz del caballo) casi todos con el monitor de equitación a la cuerda (sujetando al caballo). Al trote eran autónomos en muchas ocasiones JRS, MAGS y ERT, mientras que al paso todos podian guiar al caballo al final del programa, siendo la menos autónoma LNS que 
es la más joven y dispone de menos fuerza y desarrollo motriz". (Diario de Campo, Orientadora).

"Todos excepto LNS son capaces de mantenerse correctamente sentados y mantener el equilibrio al galope, siempre a la cuerda y guiados por una Monitora de Equitación..." (Diario de Campo, Orientadora, última sesión).

\section{Conclusiones}

A lo largo de la presente investigación llevada a cabo hemos cumplido con la gran mayoría de nuestros objetivos, pudiendo incluso adquirir evidencias teóricas y empíricas de cómo evoluciona un niño con trastorno del espectro autista en su faceta psicomotriz.

Podemos afirmar tras las observaciones de campo realizadas, que nuestra técnica (equinoterapia) es una terapia integral tanto por los logros a los que hemos llegado como por su integración y contacto con las demás terapias en las que participaba el niño en ese momento de su desarrollo. De este modo, podemos pensar que uno de los factores que posibilitan la eficacia terapéutica en este campo es el trabajo interdisciplinario que se produce entre los diferentes profesionales encargados de la terapia. Cada uno de ellos tiene una mirada diferente sobre el trastorno del niño, de modo tal que la terapia se enriquece por la participación de todas esas aportaciones, conocimientos y puntos de vista sobre el avance y diagnóstico.

La equinoterapia aporta un elemento principal que la hace única: el caballo. Este es un ser vivo de naturaleza animal que trabaja como co-terapeuta junto con el profesional de hipoterapia, uno complementa el trabajo del otro. Aquí la estimulación que reciben los niños es intensa y continua. Se realizan actividades físicas de forma lúdica, el niño al tiempo que juega y disfruta del caballo y del ambiente, sin querer y sin saber, está interactuando con otros sujetos y con el propio animal. Se establece un alto nivel de confianza no solo con el terapeuta y el caballo, sino también hacia sí mismos.

Esta terapia, a diferencia de otras, tiene un contacto más profundo con la naturaleza, en donde el lenguaje no verbal prepondera sobre el verbal. Los niños para comunicarse con el caballo no necesitan las palabras, proceden a acariciarlo, darle de comer, le miran a los ojos, se dejan oler por los caballos. El caballo para comunicarse utiliza la comunicación vocal, olfatoria, táctil.

Queda claramente reflejado que con la hipoterapia y la equitación terapéutica en el trabajo interdisciplinar con personas con autismo son múltiples los beneficios que podemos obtener en todas las áreas del desarrollo.

Entre los resultados obtenidos más destacados señalar beneficios como la mejora en el equilibrio y la postura, visibles mejoras tanto en la motricidad fina como gruesa, y una relajación muscular óptima que favorecía la eliminación paulatina de las estereotipias presentadas por nuestros usuarios.

La experiencia de trabajar con alumnos con este tipo de problemática ha sido realmente única, ya que nos ha brindado la oportunidad de compartir con ellos una gran cantidad de vivencias y situaciones novedosas, al tiempo que nos ha permitido descubrir el fascinante y a la vez difícil mundo que se despliega en este particular proceso de enseñanza-aprendizaje.

Finalmente, defender la práctica de la hipoterapia y equitación terapéutica como una muestra más de las múltiples propuestas que se pueden llevar a cabo a la hora de trabajar con niños con este tipo de trastorno, sin olvidar que estas tareas actúan sobre ellos como relajante, ya que los liberan del estrés al que se encuentran sometidos tanto física como mentalmente. Animar a otros profesionales de la Educación para que en pro de la integración de los alumnos con necesidades educativas especiales, traten de llevar estas $\mathrm{y}$ otras nuevas experiencias a sus aulas y a sus diversos proyectos de actuación.

\section{Referencias bibliográficas}

Benda, McGibbon \& Grant (2003). Improvements in muscle symmetry in children with cerebral palsy after equine-assisted therapy (hippotherapy) The journal of alternative and complimentary medicine, 9 (6), 817825.

Fine, A. H. (2006): Handbook on animal-assisted therapy: Theoretical foundations and guidelines for practice (2nd ed.). Nueva York: Academic Press.

Gross, E. (2006). Equinoterapia: la rehabilitación por medio del caballo. Sevilla. Editorial: Trillas.

Instituto Nacional de Salud Infantil y Desarrollo Humano (NICHD) (2005): Una breve introducción al autismo: lo que sabemos. Publicación del Departamento de Salud y de Servicios Humanos de los Estados Unidos, Institutos Nacionales de la Salud (NIH), 5-5592 (S). Disponible en: http://www.nichd.nih.gov/publications/pubs/upload/in troduccion_autismo_2005.pdf.

Kanner, L. (1943). Autistic disturbances of affective contact. Nervous Child, 2, 217-250.

McPhail, K. L. (2006). Occupational therapy at the barn. Evidence through measurement: Outcome measures for hippotherapy research. Hippotherapy.

Morrison, M. L. (2007): Health benefits of animalassisted interventions. Complementary Health Practice Review 12(1), 51-62.

Murphy, D. et al., Department of Physical Therapy (D.M., L.K.D.), University of Massachusetts Lowell, Lowell, Massachusetts; and EK Shriver Center of the University of Massachusetts Medical School (J.G.), Waltham, Massachusetts Pediatric Physical Therapy (2008) The Effect of Hippotherapy on Functional Outcomes for Children with Disabilities: A Pilot Study. Pediatr Phys Ther, no 20, pp. 264-270.

Snider, L., Korner-Bitensky, N., Kammann, C., Warner, S. \& Saleh, M. (2007) Horseback riding as therapy for children with cerebral palsy: Is there evidence of its effectiveness? Physical \& occupational therapy in pediatrics, $27,5-23$.

Vega, A. (2005). Autismo y Educación Física: una experiencia en el centro de día de Alfahar (Valladolid). Tándem, 19, 92-98. 